\title{
Multidimensional Unstructured-Grid Liquid Rocket Engine Nozzle Performance and Heat Transfer Analysis
}

\author{
Ten-See Wang ${ }^{*}$ \\ NASA MarshallSpace Flight Center,Huntsville,Alabama,35812
}

\begin{abstract}
The objectives of this study are to conduct a unified computational analysis for computing the design parameters such as axial thrust, convective and radiative wall heat fluxes for liquid rocket engine nozzles, so as to develop a computational strategy for computing those design parameters through parametric investigations. The computational methodology is based on a multidimensional, finite-volume, turbulent, chemically reacting, radiating, unstructured-grid, and pressure-based formulation, with grid refinement capabilities. Systematic parametric studies on effects of wall boundary conditions, combustion chemistry, radiation coupling, computational cell shape, and grid refinement were performed and assessed. Comparisons of the computed axial thrust performance, flow features, and wall heat fluxes with those of available test data and design calculations are presented.
\end{abstract}

\section{Nomenclature}

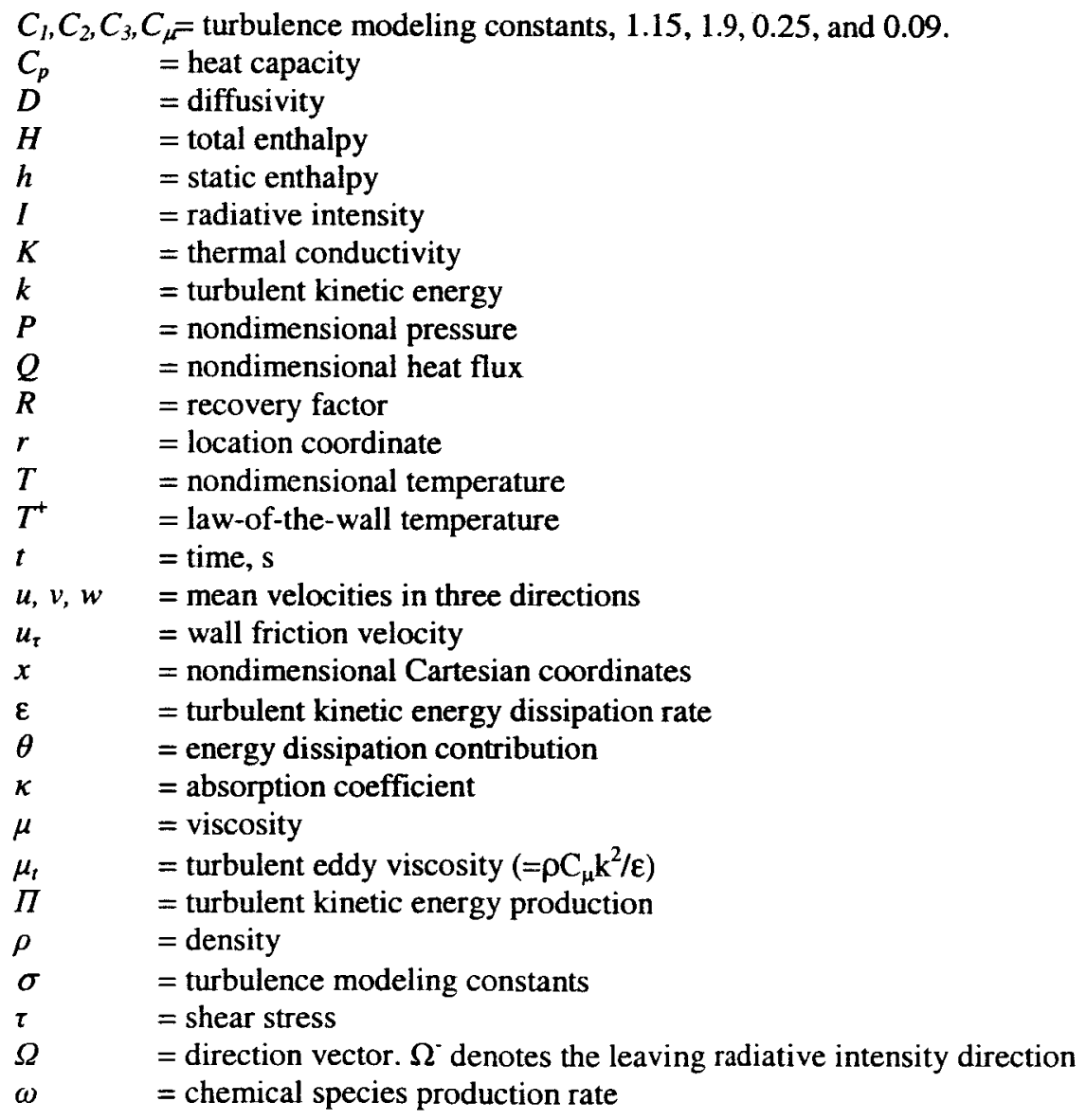

\footnotetext{
"Staff Consultant, Applied Fluid Dynamics Analysis Group, TD64, Senior Member AIAA.
} 


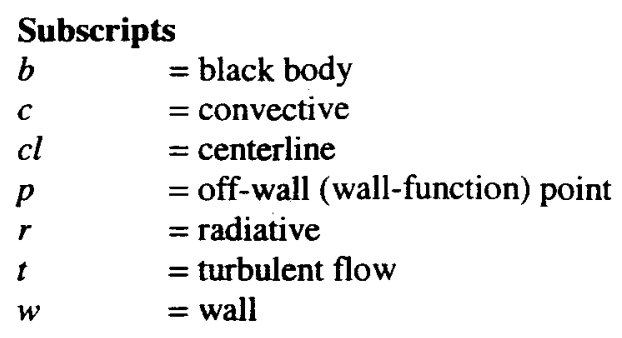

\section{Introduction}

$\mathrm{T}$

The two major factors in rocket engine design, performance and integrity (convective heat transfer), are often conducted separately. As a result, the final design based on performance may have to be altered due to convective heating considerations, and vice versa, resulting in delays and compromises. Recently, radiative heating has been generating concerns due to renewed interest in hydrocarbon engines. Those combined motivated this study to perform and demonstrate a unified analysis for the computation of those design parameters in a simultaneous fashion. Systematic parametric studies on effects of wall boundary conditions, combustion chemistry, radiation coupling, computational cell shape, and grid refinement were performed and assessed, in order to come up with a strategy for efficient and realistic computations.

A nozzle axial force analysis, ${ }^{1}$ and a conjugate convective heat transfer analysis ${ }^{2}$ for axisymmetric Space Shuttle Main Engine (SSME) thruster were reported in the 1990's, using a structured-grid, multi-zone computational fluid dynamics (CFD) solver FDNS. As the requirements for parallel computing efficiency and faster grid generation arise, an unstructured-grid CFD methodology UNIC was developed recently through several activities, namely the launch vehicle base-heating, ${ }^{3}$ Laser propulsion, ${ }^{4}$ and stage-separation. ${ }^{5}$ This unstructured-grid CFD methodology is refined in this study to conduct the unified axial force, convective and radiative heat transfer analyses on the SSME thruster flowfield, simulating the hot-firing operation at sea level. Both axisymmetric and three-dimensional analyses were performed and a final computational strategy reported.

\section{Computational Methodology}

The time-varying transport equations of continuity, species continuity, momentum, global energy (total enthalpy), turbulent kinetic energy, and turbulent kinetic energy dissipation can be written as:

$$
\begin{aligned}
& \frac{\partial \rho}{\partial t}+\frac{\partial}{\partial x_{j}}\left(\rho u_{j}\right)=0 \\
& \frac{\partial \rho \alpha_{i}}{\partial t}+\frac{\partial}{\partial x_{j}}\left(\rho u_{j} \alpha_{j}\right)=\frac{\partial}{\partial x_{j}}\left[\left(\rho D+\frac{\mu_{t}}{\sigma_{\alpha}}\right) \frac{\partial \alpha_{i}}{\partial x_{j}}\right]+\omega_{i} \\
& \frac{\partial \rho u_{i}}{\partial t}+\frac{\partial}{\partial x_{j}}\left(\rho u_{j} u_{i}\right)=-\frac{\partial p}{\partial x_{i}}+\frac{\partial \tau_{i j}}{\partial x_{j}} \\
& \frac{\partial \rho H}{\partial t}+\frac{\partial}{\partial x_{j}}\left(\rho u_{j} H\right)=\frac{\partial p}{\partial t}+Q_{r}+\frac{\partial}{\partial x_{j}}\left(\left(\frac{K}{C_{p}}+\frac{\mu_{i}}{\sigma_{H}}\right) \nabla H\right) \\
& \quad+\frac{\partial}{\partial x_{j}}\left(\left(\left(\mu+\mu_{t}\right)-\left(\frac{K}{C_{p}}+\frac{\mu_{i}}{\sigma_{H}}\right)\right) \nabla\left(V^{2} / 2\right)\right)+\theta \\
& \frac{\partial \rho k}{\partial t}+\frac{\partial}{\partial x_{j}}\left(\rho u_{j} k\right)=\frac{\partial}{\partial x_{j}}\left[\left(\mu+\frac{\mu_{t}}{\sigma_{k}}\right) \frac{\partial k}{\partial x_{j}}\right]+\rho(\Pi-\varepsilon) \\
& \frac{\partial \rho \varepsilon}{\partial t}+\frac{\partial}{\partial x_{j}}\left(\rho u_{j} \varepsilon\right)=\frac{\partial}{\partial x_{j}}\left[\left(\mu+\frac{\mu_{t}}{\sigma_{\varepsilon}}\right) \frac{\partial \varepsilon}{\partial x_{j}}\right]+\rho \frac{\varepsilon}{k}\left(C_{1} \Pi-C_{2} \varepsilon+C_{3} \Pi^{2} / \varepsilon\right)
\end{aligned}
$$


where the energy dissipation contribution $\theta$ can be expressed as:

$$
\theta=\frac{\partial}{\partial x_{i}}\left[\left(\mu+\mu_{t}\right)\left(u_{j} \frac{\partial u_{i}}{\partial x_{j}}-(2 / 3) u_{i} \frac{\partial u_{j}}{\partial x_{j}}\right)\right]
$$

and the shear stress $\tau_{i j}$ can be expressed as:

$$
\tau_{i j}=\left(\mu+\mu_{t}\right)\left(\frac{\partial u_{i}}{\partial x_{j}}+\frac{\partial u_{j}}{\partial x_{i}}-\frac{2}{3} \frac{\partial u_{k}}{\partial x_{k}} \delta_{i j}\right)
$$

A predictor and corrector solution algorithm was employed to provide coupling of the fluid governing equations. A second-order central-difference scheme was employed to discretize the diffusion fluxes and source terms of the governing equations. For the convective terms, a second-order total variation diminishing difference scheme was used in this effort. To enhance the temporal accuracy, a second-order backward difference scheme was employed to discretize the temporal terms. A point-implicit (operator splitting) method was employed to solve the chemistry system.

An extended $k-\varepsilon$ turbulence model ${ }^{6}$ was used to describe the turbulence. A 7-species, 9-reaction detailed mechanism ${ }^{7}$ was used to describe the finite-rate, hydrogen/oxygen $\left(\mathrm{H}_{2} / \mathrm{O}_{2}\right)$ afterburning chemical kinetics. The seven species are $\mathrm{H}_{2}, \mathrm{O}_{2}, \mathrm{H}_{2} \mathrm{O}, \mathrm{O}, \mathrm{H}, \mathrm{OH}$, and $\mathrm{N}_{2}$.

A modified wall function approach was employed to provide wall boundary layer solutions that are less sensitive to the near-wall grid spacing. Consequently, the model has combined the advantages of both the integrated-to-thewall approach and the conventional law-of-the-wall approach by incorporating a complete velocity profile and a universal temperature profile ${ }^{7}$. This approach is especially useful in three-dimensional (3-D) applications.

The convective heat transfer follows the modified Newtonian law

$$
\left.Q_{c}=\left(\rho u_{\tau} / T^{+}\right) h_{h_{w}}-h_{p}-R\left(u_{p}^{2} / 2\right)\right)
$$

The radiative heat transfer is analyzed by solving the radiative transfer equation

$$
(\Omega \cdot \nabla) I(r, \Omega)=-\kappa I(r, \Omega)+\kappa I_{b}(r)
$$

Discrete ordinate method was used to solve the radiative transfer equation and $\mathrm{H}_{2} \mathrm{O}$ is the major radiating medium. The spectral-line based weighted-sum-of-gray-gases model ${ }^{8}$ was used to calculate the total emissivity and absorptivity of the radiating medium. Details of the numerical algorithm can be found in Ref's 3-5. The radiative heat flux is given by the integration of the wall leaving radiative intensities

$$
Q_{r}=\int_{n \cdot \Omega^{-}<0} I\left(r, \Omega^{-}\right)\left|n \Omega^{-}\right| d \Omega^{-}
$$

\section{Computational Grid Generation}

The flowfields of four axisymmetric and four 3-D grids were computed during the course of the study. The results from two representative axisymmetric and two 3-D grids are reported for conciseness. These grids are hybrid grids and can be classified into two groups: the structure-grid dominated and the unstructured-grid dominated. Figure 1 shows the layout of an unstructured-grid dominated hybrid grid ax 1 , which is an axisymmetric grid and has 4 layers of structured (quadrilateral) grid surrounding the solid walls, while the rest of the domain filled with unstructured (triangular) cells. These structured-grid layers are used to ensure proper wall boundary layer development. The layout of a structure-cell dominated hybrid grid ax6 is shown in Fig. 2. Axisymmetric grid ax6 has the interior of the thruster and plume region filled with quadrilateral cells, while the rest of the domain filled with triangular cells. The structured-grid layers used in grid ax1 are also embedded in grid ax6, and in 3-D grids $3 \mathrm{~d} 6$ and $3 \mathrm{~d} 9$ (Figs 3-4), such that the boundary layer development for all grids is similar. Figure 3 shows the layout of the hybrid 3-D grid 3d6. It was constructed by rotating grid ax6 72 times for 360 degrees. Figure 4 shows the 
layout of the hybrid grid $3 \mathrm{~d} 9$. These computational grids were generated using the software package GRIDGEN. 9 Table 1 shows the total number of points and cells in these four grids. The structured cells in grids $3 \mathrm{~d} 6$ and $3 \mathrm{~d} 9$ are hexahedral elements. The unstructured cells in grid $3 \mathrm{~d} 9$ are tetrahedral elements, while the unstructured cells in grid $3 \mathrm{~d} 6$ are prismatic elements. Note that all 4 grids were computed as a single zone, thus avoiding the interface complexities commonly seen in multi-zonal grids.

Table 1. Number of points and cells of the axisymmetric and 3-D grids.

\begin{tabular}{|l|r|r|r|r|}
\hline Grid & \# points & \# cells & $\begin{array}{r}\text { \# structured } \\
\text { cells }\end{array}$ & $\begin{array}{r}\text { \# unstructured } \\
\text { cells }\end{array}$ \\
\hline Ax1 & 17,509 & 30,578 & 2,016 & 28,562 \\
\hline Ax6 & 17,391 & 17,710 & 15,300 & 2,410 \\
\hline 3d6 & $1,286,934$ & $1,275,120$ & $1,101,600$ & 173,520 \\
\hline 3d9 & 418,165 & $1,732,081$ & 227,984 & $1,504,097$ \\
\hline
\end{tabular}

\section{Boundary Conditions and Run Matrix}

Fixed total condition was used for the inlet of the thruster and the outer boundary. A total pressure of 1 atm was specified for the outer boundary in order to simulate the nozzle hot-firing at sea level. No-slip boundary condition was specified for the thruster walls. Symmetry condition was applied to the centerline for axisymmetric cases. The CEC program ${ }^{10}$ was used to obtain the chamber equilibrium species composition for use at the thruster inlet.

The run matrix is shown in Table 2 . These cases were built up systematically in order to understand the grid effects such as cell shape and grid refinement, and the modeling effects such as wall boundary condition, chemistry, and radiation. For the convenience of presentation, abbreviated letters are used to represent different cases in the run matrix. For example, case $\mathrm{f} z$ represents parametric conditions of adiabatic wall and frozen chemistry, while case frcgr uses parametric conditions of cooled wall and finite-rate chemistry, with radiation coupling and grid refinement turned on. Due to the limitation of current resources, grid refinement was not preformed for the 3-D cases.

Table 2. Run matrix

\begin{tabular}{|l|l|l|l|l|}
\hline Case & wall & chemistry & $\begin{array}{l}\text { Grid } \\
\text { refinement }\end{array}$ & Radiation \\
\hline fz & adiabatic & frozen & no & no \\
\hline eq & adiabatic & equilibrium & no & no \\
\hline fr & adiabatic & Finite-rate & no & no \\
\hline frc & cooled & Finite-rate & no & no \\
\hline frcr & cooled & Finite-rate & no & yes \\
\hline frcg & cooled & Finite-rate & yes & no \\
\hline frcgr & cooled & Finite-rate & yes & yes \\
\hline
\end{tabular}

\section{Results and Discussion}

The computation was performed on a cluster machine using 4 processors for each axisymmetric case and 32 processors for each 3-D case. A global time step of $1 \mu \mathrm{s}$ was used. Figure 5 shows a comparison of the computed adiabatic wall temperatures for grid ax6. Similar, if not identical wall temperature profiles were obtained for grid axl and are not shown. The computed wall temperature for the frozen chemistry case is constant, indicating the conservation laws are satisfied. Those for the equilibrium and finite-rate chemistry cases increase first after the throat, due to the recombination of chemical species to become $\mathrm{H}_{2} \mathrm{O}$. The temperatures for those two cases then decrease as $\mathrm{H}_{2} \mathrm{O}$ dissociates. Of interest is the temperature for the equilibrium case, it drops continuously until it closes to that of the frozen flow, near the nozzle exit. This is expected since the stagnation temperature is very close to the chamber temperature with which the frozen composition was determined with an equilibrium solution. This also implies the equilibrium chemistry probably dissociates the $\mathrm{H}_{2} \mathrm{O}$ at too fast a rate inside the nozzle. A specified cooled wall temperature profile ${ }^{1}$ is also shown in Fig. 5 which was determined through a separate conjugate heat transfer calculation; this temperature profile is used later as a boundary condition to consider the effect of heat loss to the regenerative coolant channels. 
Figure 6 shows the computed Mach number contours for cases $\mathrm{fz}$ and frcgr for grids ax1 and ax6, respectively; those for other cases are similar to those of case frcgr and are not shown. These figures show the captured nozzle flow features (nozzle shock, lip shock, triple point, Mach disc, shock reflection, and shear layer/shock interaction). In general, all cases capture the flow features reasonably well, except the frozen flow case in which a curved Mach disk was obtained. It can also be seen that the nozzle shocks appear to be sharper in the contours of the structuredcell dominated grid ax6 than those of the unstructured-element dominated grid ax1. The sharpest nozzle flow features are captured with grid refinement on grid ax6, while the added radiation changes the flow features only slightly.

The significance of a curved disk is that a large flow recirculation appears behind the curved disk. The occurrence of the curved disk may be attributed to the difference in thermodynamics between the frozen flow and chemically reacting flows. As shown in the centerline $\mathrm{H}_{2} \mathrm{O}$ mass fraction, specific heat ratio and Mach number profiles in Fig. 7, the inability of recombining the species of the frozen flow results in much higher specific heat ratios than those of the reacting flows, which in turn produces high Mach numbers along the centerline. The higher shock strength leads to higher total pressure loss across the shock, which causes the shock center to retreat and consequently an overall curved disk. On the other hand, the curves of equilibrium chemistry closely follow those of finite-rate chemistry. This is because the centerline temperatures drop continuously and are much lower than the chamber temperature (Fig. 8), hence the dissociation process occurring on the adiabatic wall is frozen on the centerline. Furthermore, the curved disk phenomenon happens both in grid ax1 and ax6, hence it is cell-shape independent and thermodynamics induced.

Figure 8 shows a comparison of the thruster centerline temperatures for grid ax6. The frozen chemistry gives the lowest bound while all other cases group together as an upper bound, while the result from Ref. 1 falls in between. As discussed above, the low frozen chemistry curve is caused by the thermodynamics. As for the result from Ref. 1, it is speculated that an older thermodynamics database was used then. A comparison of the thruster wall pressures is shown in Fig. 9. The computed results from all cases appear to group together and agree reasonably well with the test data. Figure 10 shows a comparison of thruster centerline pressures. All predictions agree reasonably well, except for the frozen flow case that deviates lower near the nozzle lip.

Figure 11 shows the computed convective heat fluxes for grid ax6. As expected, the peak convective heat fluxes occur at the throat $(x=0)$ for all cases. The refined grid gives a slightly lower peak heat flux. Radiation does not affect the convective heat flux, because the maximum radiative heat flux is about two orders-of-magnitude lower than that of convection (Fig. 12). All predictions compare reasonably well with those of the three design methods. ${ }^{2,11}$ Result from Fig. 11 demonstrates that the wall boundary layers were captured reasonably well. The difference in the initial heat fluxes is caused by the difference in ways of initiating the boundary layers among different methods and the significance of which is negligible in comparison to the peak heat flux.

Figure 12 shows the computed radiative heat flux for grid ax6 while cooled wall, finite-rate chemistry, and grid refinement were used as operating conditions. As expected, high radiative heating occurs inside the combustion chamber within which the high temperature and high $\mathrm{H}_{2} \mathrm{O}$ concentration are prevalent. As the propulsive flow expands past the throat, the temperature drops, hence the low radiative heat flux. The peak radiative heat flux is about two orders of magnitude lower than that of the convective heat flux, which is reasonable for a hydrogen fueled engine. In current methodology, the injector faceplate is modeled as a black body. In order to compare the predicted radiation with that of a plume radiation code GASRAD, ${ }^{12}$ which does not model the injector faceplate, another run was performed by setting the temperature of the injector faceplate to $300 \mathrm{deg}$. K, effectively turning off the black body radiation. The structured-grid solution from Ref. 1 was used as input for GASRAD radiation calculation, since GASRAD can not read unstructured-grid information. It can be seen that the result from turning off the blackbody radiation at the inlet, using a weighted-sum-of-gray-gases absorption model, compares reasonably well with that of GASRAD in which a narrow band absorption model was used. It should be pointed out that GASRAD reads in flow solution for a decoupled radiation solution, and current methodology solves the flow equations and radiative transfer equation simultaneously. The computed peak value is higher when the black body radiation is included at the inlet, as expected. It is also noted that GASRAD was developed for the prediction of plume radiation, hence it does not consider the re-radiation from the solid walls. In addition, it solves the line-ofsight equation and not the radiative transport equation.

Table 4. Comparison of SSME thrust chamber specific impulses (ISP's) for axisymmetric cases

\begin{tabular}{|l|c|c|}
\hline & Ax1 & Ax6 \\
\hline $\mathrm{fz}$ & 438.7 & 439.7 \\
\hline $\mathrm{eq}$ & 455.6 & 456.0 \\
\hline $\mathrm{fr}$ & 455.2 & 455.6 \\
\hline
\end{tabular}




\begin{tabular}{|l|l|l|}
\hline frc & 452.4 & 452.6 \\
\hline frcg & 452.9 & 453.7 \\
\hline frcgr & 452.5 & 453.3 \\
\hline Data & \multicolumn{2}{|c|}{453.3} \\
\hline
\end{tabular}

Table 4 shows the comparison of computed SSME thrust chamber specific impulses, or the axial thrust performances for the axisymmetric cases. The frozen flow calculations give too low an axial force, even for the adiabatic wall assumption that assumes zero wall heat loss. This is again caused by inadequate heat capacity distributions forced by a fixed species composition. The reacting flow (with adiabatic wall) cases overpredict the data for about 2 3 s, with the equilibrium case giving the highest values. When the wall heat loss is considered, the axial force predictions become very close to the data. The quadrilateral cell dominated grid ax6 appears to predict slightly better ISP's than those of the triangular cell dominated grid ax1. Within grid ax6, the grid refinement and radiation options offer the best agreement.

Figure 13 shows the computed temperature contours for grid 3d6, case frc. Similar to the Mach number contours, the temperature contours also show the captured nozzle flow physics such as the nozzle shock, lip shock, triple point, Mach disc, shock reflection, and shear layer/shock interaction. Two perpendicular planes are used to give the Mach disc a three-dimensional feel. The high temperature in the mixing layer indicates after burning.

Figure 14 shows a comparison of computed thruster centerline temperatures. The centerline temperature of grid $3 \mathrm{~d} 6$ matches that of grid ax6 reasonable well, except inside the chamber where the temperature of grid $3 \mathrm{~d} 6$ is slightly lower. Figure 15 compares the wall pressures. The wall pressures of grid $3 \mathrm{~d} 6$ and ax 6 overlap and both compare reasonably well with the data. Figure 16 compares the centerline pressures. The centerline pressure of grid $3 \mathrm{~d} 6$ coincides with that of grid ax6, until the nozzle lip where the pressure of grid ax6 is slightly higher.

Figure 17 shows a comparison of convective wall heat fluxes. The computed heat fluxes agree reasonably well with those of the design methods. The predictions for grid $3 \mathrm{~d} 6$ overlap with those of grid ax6. The radiation does not affect the convective heat fluxes of grid $3 \mathrm{~d} 6$, again due to the relative low radiative heat fluxes inside a $\mathrm{H}_{2} / \mathrm{O}_{2}$ engine. Figure 18 shows a comparison of the computed radiative wall heat fluxes. Similar to the result of the axisymmetric cases (Fig. 12), the computed radiative fluxes using a weighted-sum-of-gray gases absorption model compares reasonably well with that of GASRAD using a narrow band absorption model, while turning off the blackbody radiation at the inlet. And the predicted radiative heat flux is higher while turning on the blackbody radiation at the inlet.

Table 5. Comparison of SSME thrust chamber specific impulses (ISP's) for 3-D cases

\begin{tabular}{|l|c|c|}
\hline & $3 \mathrm{~d} 6$ & $3 \mathrm{~d} 9$ \\
\hline $\mathrm{fz}$ & 439.6 & 436.5 \\
\hline eq & 456.4 & 453.3 \\
\hline $\mathrm{fr}$ & 454.9 & 452.5 \\
\hline frc & 453.2 & 450.0 \\
\hline frcr & 453.1 & 449.9 \\
\hline Data & \multicolumn{2}{|c|}{453.3} \\
\hline
\end{tabular}

Table 5 shows the comparison of computed specific impulses for the 3-D cases. The qualitative trend among the cases is very similar to the corresponding axisymmetric cases. The results from grid $3 \mathrm{~d} 9$ are consistently lower than those of grid 3d6. This is because the effective cell density of grid 3d9 is less than that of 3d6, although the total number of cells in grid $3 \mathrm{~d} 9$ is higher than that of grid $3 \mathrm{~d} 6$ (Table 1). As a general rule of thumb, the accuracy of two tetrahedral cells is approximately equivalent to that of one hexagonal cell. On the other hand, since the number of cells in grid $3 \mathrm{~d} 9$ is more than those in grid $3 \mathrm{~d} 6$, it costs more to run grid $3 \mathrm{~d} 9$. This demonstrates that the structuredcell dominated grid 3d6 is favorable both in terms of accuracy and computational efficiency, similar to the findings in the axisymmetric cases. This also agrees with the result of Huynh's Fourier analysis ${ }^{13}$ that the upwind scheme prefers structured meshes. Within grid 3d6, again the result of case frc compares very well with that of the measurement, while the addition of radiation (case frcr) changes the value only slightly.

\section{Conclusions}

Unified computational analyses for computing the design parameters such as the axial thrust, convective and radiative wall heat fluxes for liquid rocket engine thrusters were conducted, in order to develop a computational 
strategy for computing those design parameters through parametric investigations. The computational methodology is based on a multi-dimensional, finite-volume, turbulent, chemically reacting, radiating, unstructured-grid, and pressure-based formulation. Systematic parametric studies on effects of wall boundary conditions, combustion chemistry, radiation coupling, computational cell shape, and grid refinement were performed and assessed. Under the computational framework of this study, it is found that the structured-mesh performed favorably than the unstructured-mesh. The effects of radiation coupling and grid refinement were demonstrated. Finite-rate chemistry performed favorably than the equilibrium chemistry, while the frozen chemistry is undesirable, due to thermodynamics considerations. For regeneratively cooled engines, incorporating the effect of heat loss drastically improves the axial force predictions. The computed flow physics, axial thrust performance, and wall heat fluxes compared well with those of available test data and design calculations, when the favored computational strategy was used.

\section{Acknowledgments}

This study was partially supported by the Program Support and Integration project and the Generation II Launch Vehicle - Stage Separation project. The numerical platform was originally developed by Engineering Sciences, Inc. for vehicle base-heating applications, under a Small Business Innovative Research Phase II contract. Discussions with Warner Dahm resulted in the improved energy equation implementation and those with Jeff West resulted in the improved point implicit method implementation. Francisco Canabal and Alan Droege made suggestions in grid generation. Young-Ching Lee performed the GASRAD radiation calculation. Discussions with Yen-Sen Chen of Engineering Sciences, Inc. on shock wave physics, and Jiwen Liu of Taiteck and John Reardon of Remtech on radiation physics are also acknowledged.

\section{References}

${ }^{1}$ Wang, T.-S. and Chen Y.-S., "Unified Navier-Stokes Flowfield and Performance Analysis of Liquid Rocket Engines," Journal of Propulsion and Power, Vol. 9, No. 5, Sept.-Oct. 1993, pp.678-685.

${ }^{2}$ Wang, T.-S., and Luong, V., "Hot-Gas-Side and Coolant-Side Heat Transfer in Liquid Rocket Engine Combustors," Journal of Thermophysics and Heat Transfer, Vol. 8, No. 3, July-Sept. 1994, pp.524-530.

${ }^{3}$ Chen, Y.-S., Liu, J., Zhang, S., and Mallapragada, P., “An Integrated Tool for Launch Vehicle Base-Heating Analysis," Final Report, Engineering Sciences, Inc., Huntsville, AL, December, 2001.

${ }^{4}$ Wang, T.-S., Chen, Y.-S., Liu, J., Myrabo, L.N., and Mead, F.B. Jr., “Advanced Performance Modeling of Experimental Laser Lightcraft," Journal of Propulsion and Power, Vol. 18, No. 6, November-December, 2002, pp. 1129-1138.

${ }^{5}$ Chen. Y.-S., Zhang S., and Liu, J., "Stage Separation Performance Analysis Project," Final Report, Engineering Sciences, Inc., Huntsville, AL, June, 2002.

${ }^{6}$ Chen, Y.-S., and Kim, S. W., "Computation of Turbulent Flows Using an Extended k- $\varepsilon$ Turbulence Closure Model," NASA CR-179204, Oct. 1987.

${ }^{7}$ Wang, T.-S., Droege, A., D’Agostino, M., Lee, Y.-C., and Williams, R., "Base-Bleed Effect on X-33 Aerospike Plume Induced Base-Heating Environment During Power-Pack Out," AIAA Paper 2003-3651, June 2003.

${ }^{8}$ Liu, J., Shang, H.-M., Chen, Y.-S., and Wang, T.-S., "GRASP: A General Radiation Simulation Program," AIAA Paper $97-$ 2559, June, 1997.

${ }^{9}$ Steinbrenner, J.P., Chawner, J.R., and Fouts, C., "Multiple Block Grid Generation in the interactive Environment," AIAA Paper 90-1602, June 1990.

${ }^{10}$ Svehla, R.A., and McBride, B.J., "FORTRAN IV Computer Program for Calculation of Thermodynamic and Transport Properties of Complex Chemical Systems," NASA TN D-7056, Jan. 1973.

${ }^{11}$ Naraghi, M.H.N., "RTE - A Computer Code for Three-Dimensional Rocket Thermal Evaluation," User Manual, Mechanical Engineering, Manhattan College, Riverdale, NY, 2002.

${ }^{12}$ Reardon, J., and Lee, Y.-C., "A Computer Program for Thermal Radiation from Gaseous Rocket Plumes (GASRAD)," Report RTR 014-9, REMTech, Inc., Huntsville, AL, Dec. 1979.

${ }^{13}$ Huynh, H.T., "Analysis and Improvement of Upwind and Centered Schemes on Quadrilateral and Triangular Meshes," AIAA Paper 2003-3541, June 2003. 


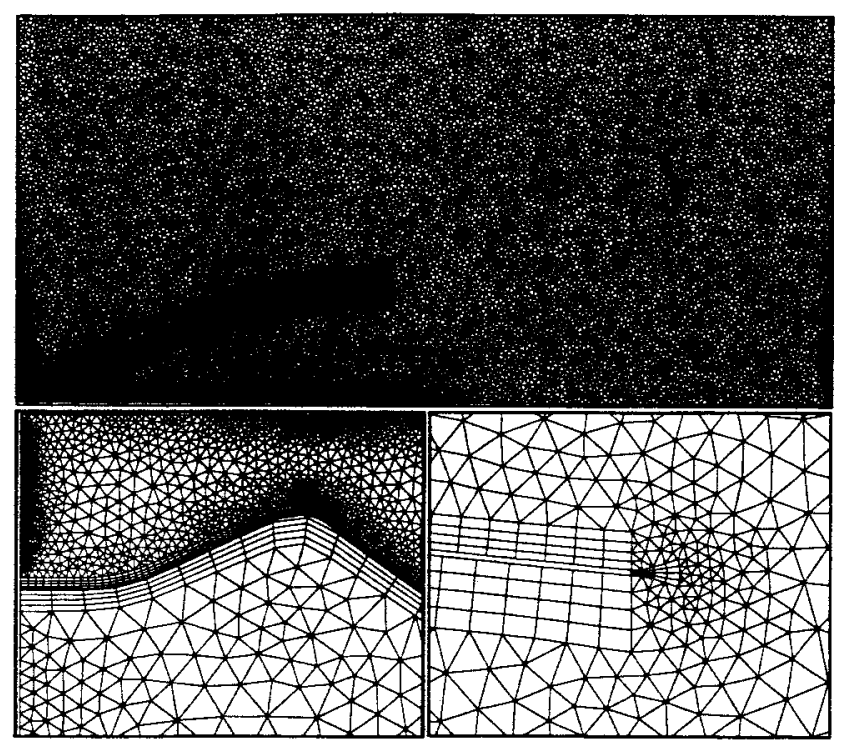

Fig. 1 The layout of hybrid grid ax1. Top: the overall grid. Bottom left: close-up near the throat. Bottom right: close-up near the nozzle lip.

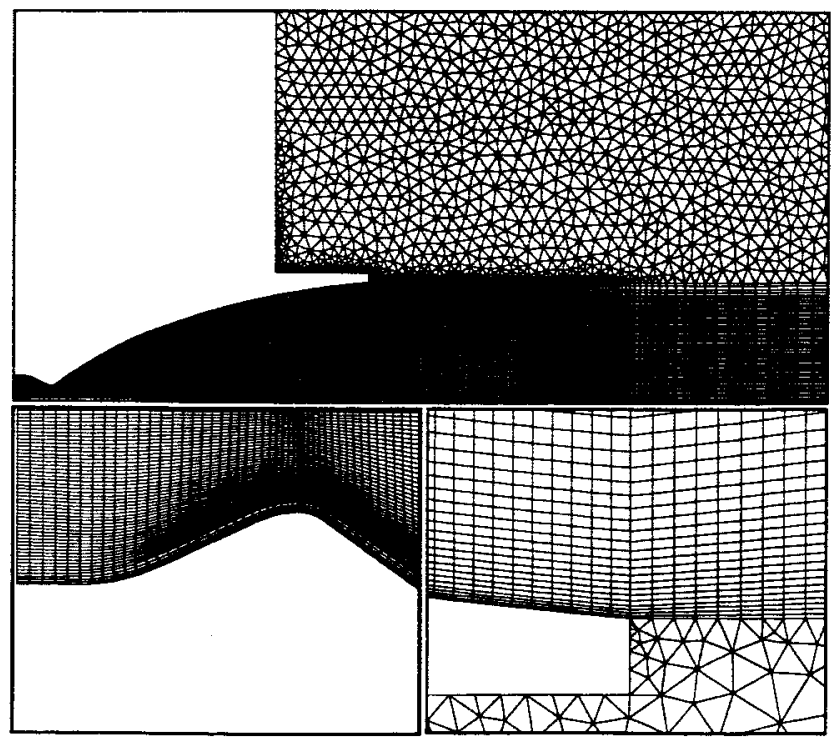

Fig. 2 The layout of hybrid grid ax6. Top: the overall grid. Bottom left: close-up near the throat. Bottom right: close-up near the nozzle lip.

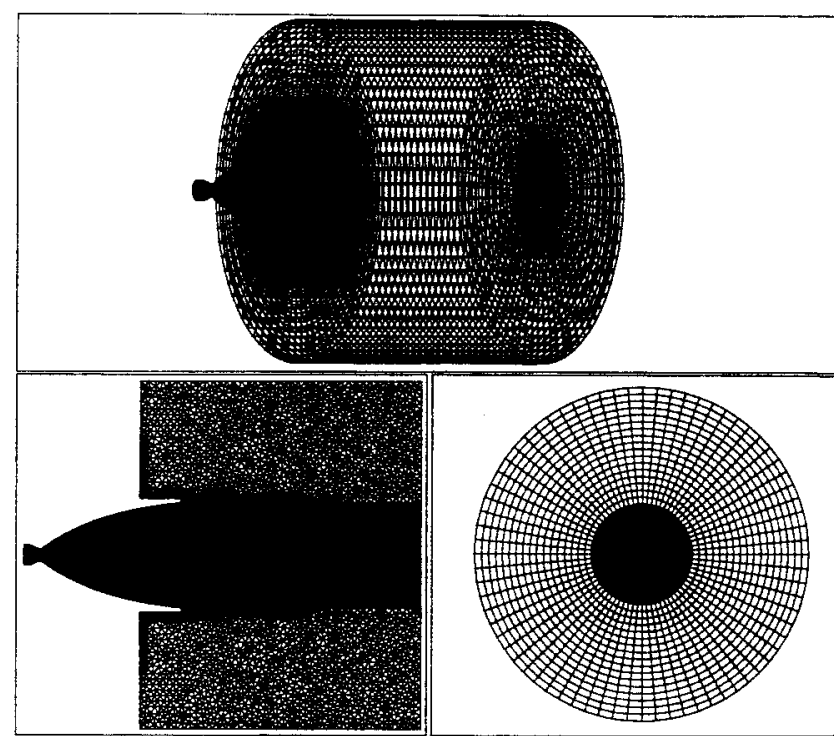

Fig. 3 Layout of hybrid grid 3d6. Upper figure: an overall view. Lower left: a cross-sectional cut through the axis. Lower right: the exit plane.

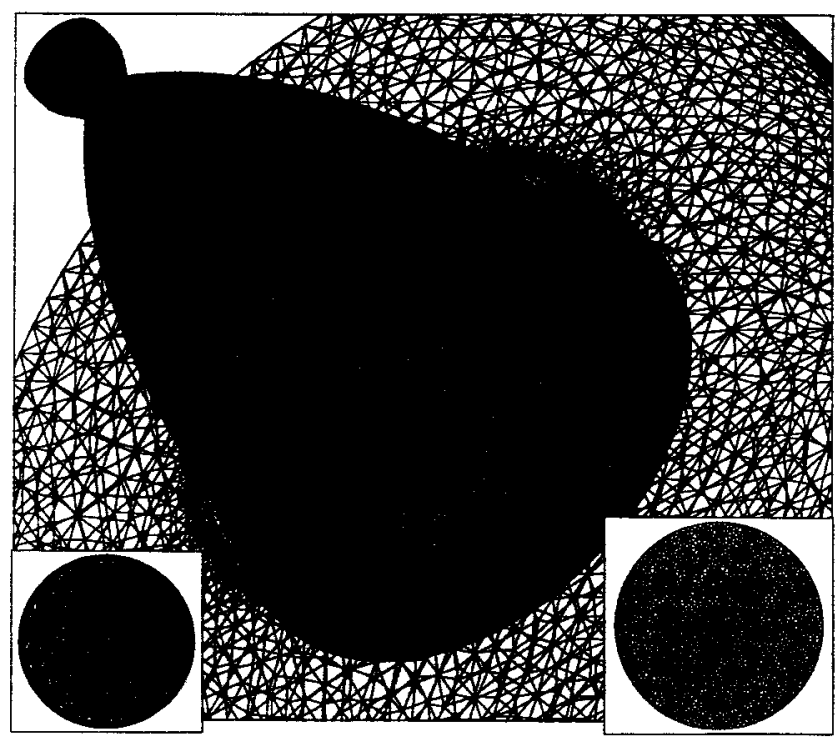

Fig. 4 Layout of hybrid grid 3d9. Lower left insert: the cross-sectional cut of the thruster inlet. Lower right insert: the cross-sectional cut of the exit plane. 


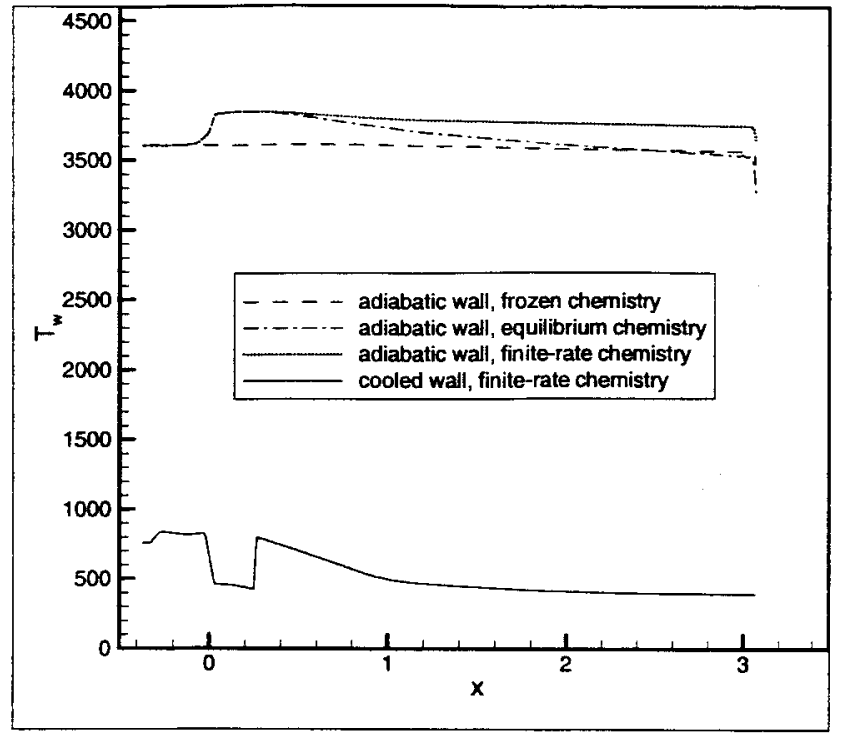

Fig. 5 A comparison of computed and specified regeneratively cooled wall temperatures for grid ax6.

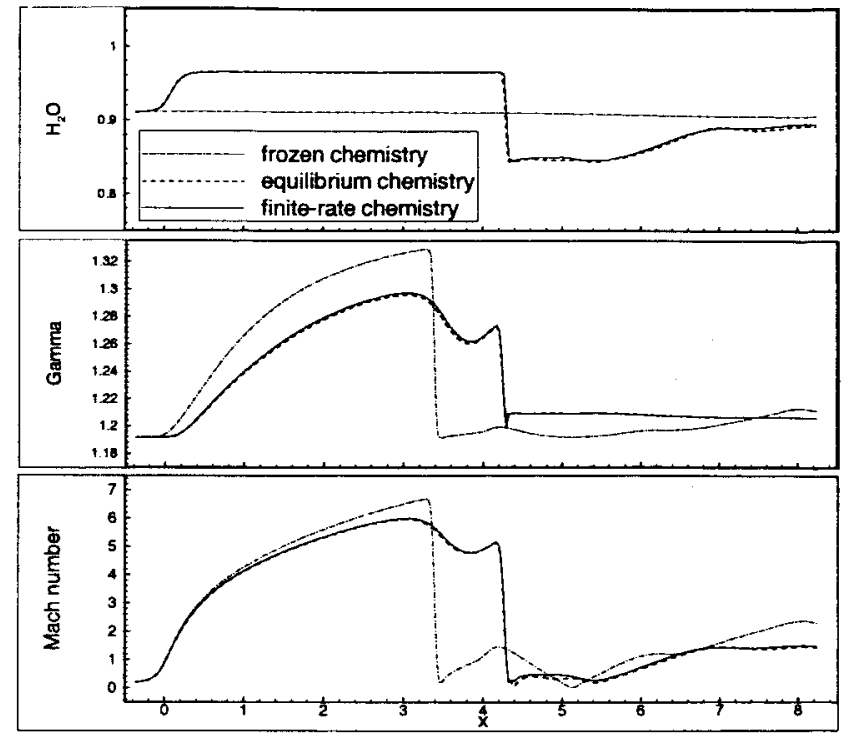

Fig. 7 A comparison of computed centerline $\mathrm{H}_{2} \mathrm{O}$ mass fractions, specific heats and Mach numbers for grid ax6.

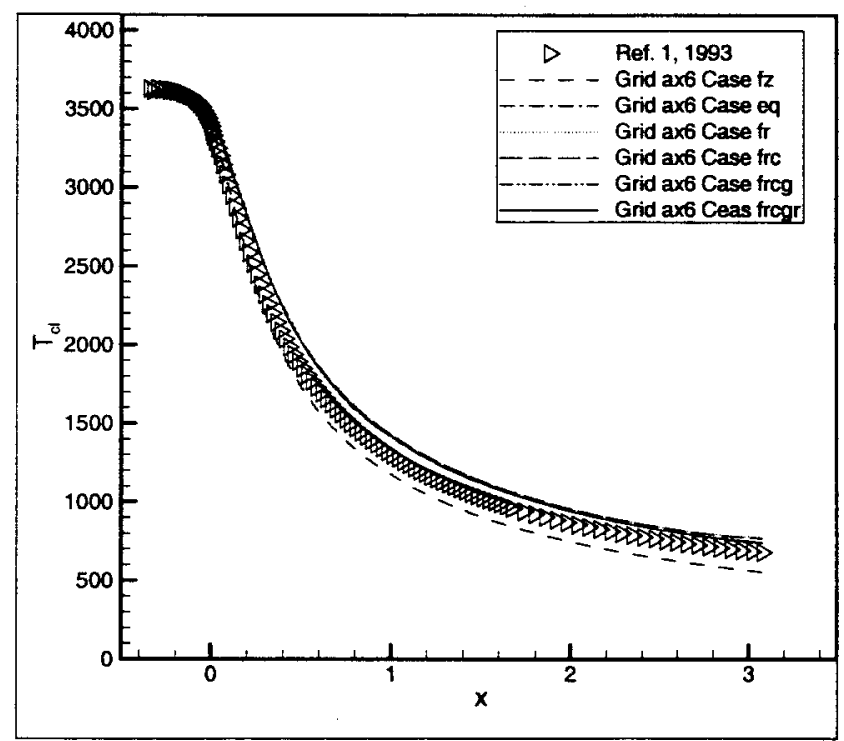

Fig. 8 A comparison of thruster centerline temperatures for grid ax6.

Fig. 6 Computed Mach number contours. a) case $f$, and d) case frcgr, grid ax6.

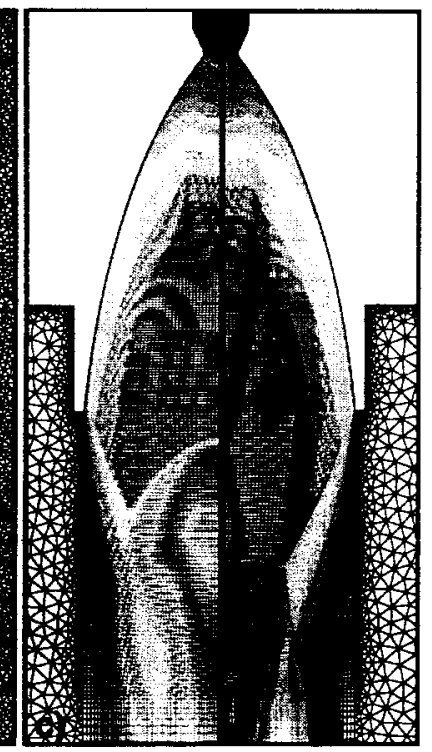




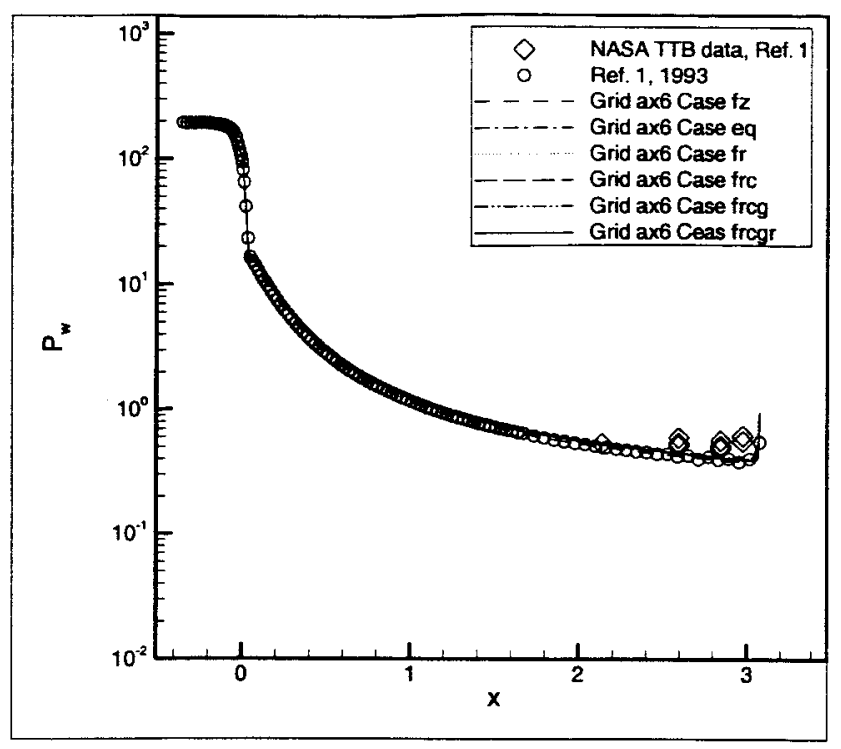

Fig. 9 A comparison of thruster wall pressures for grid ax6.

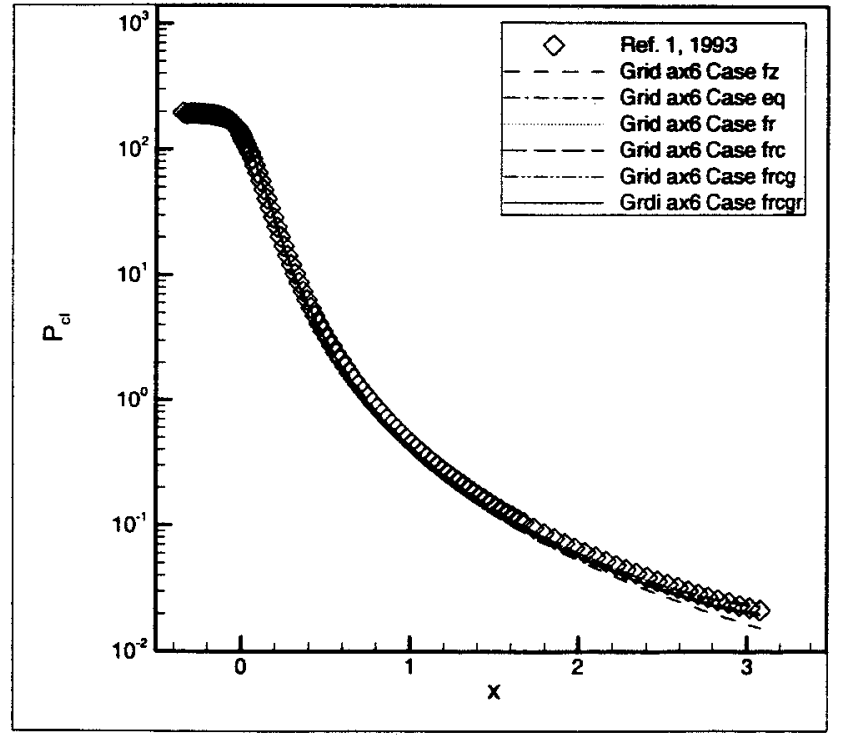

Fig. 10 A comparison of thruster centerline pressures for grid ax6.

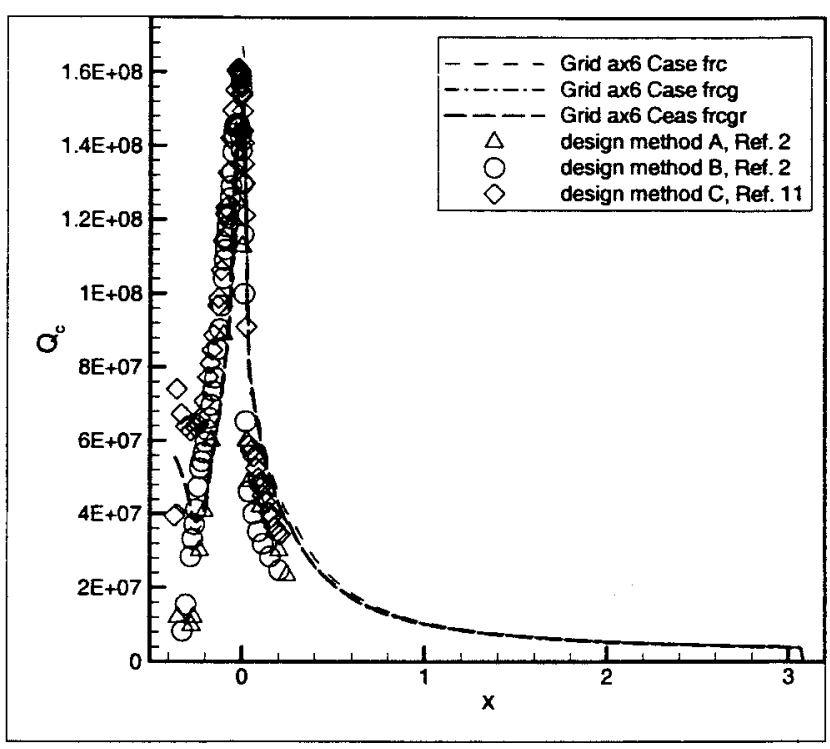

Fig. 11 A comparison of wall heat fluxes for grid ax6.

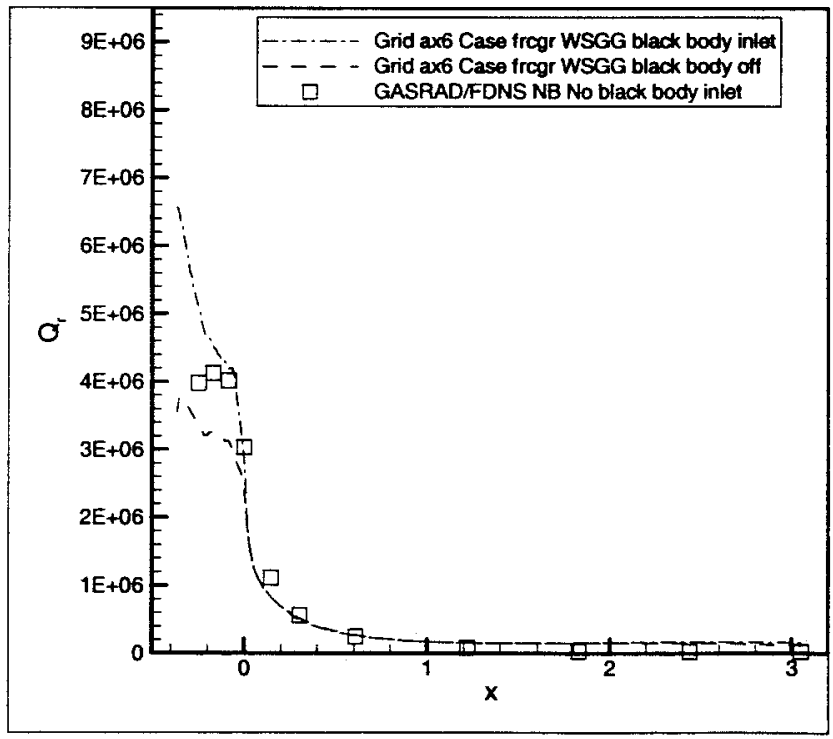

Fig. 12 A comparison of radiative wall heat fluxes for grid ax6. 


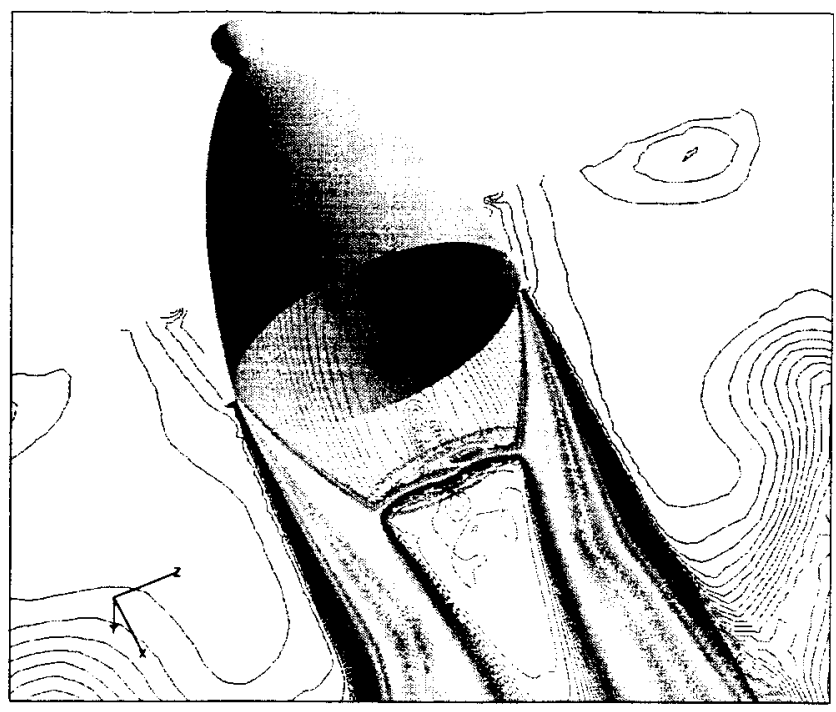

Fig. 13 Computed temperature contours for grid 3d6, case frc.

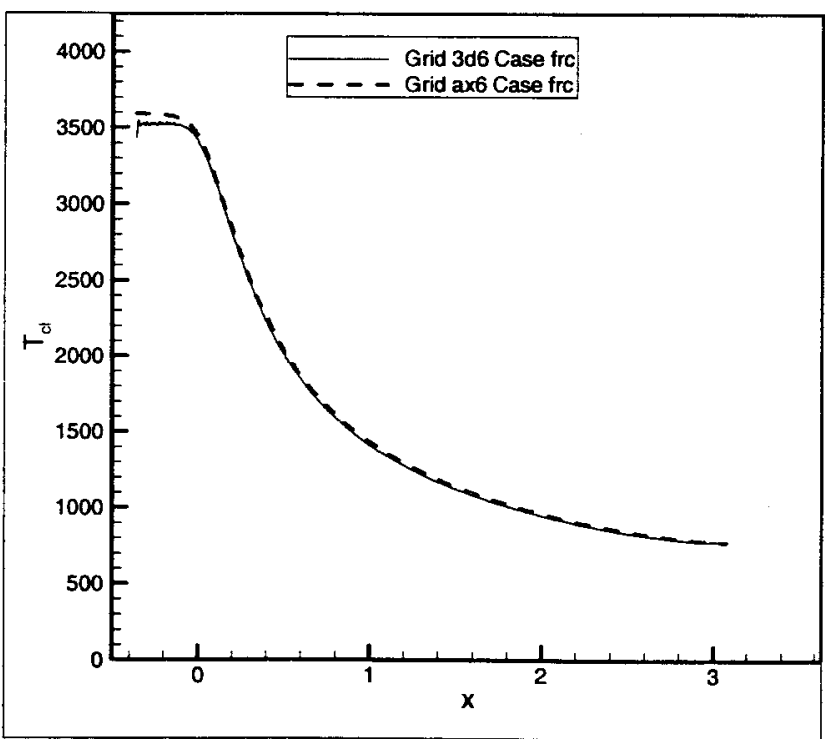

Fig. 14 A comparison of thruster centerline temperatures.

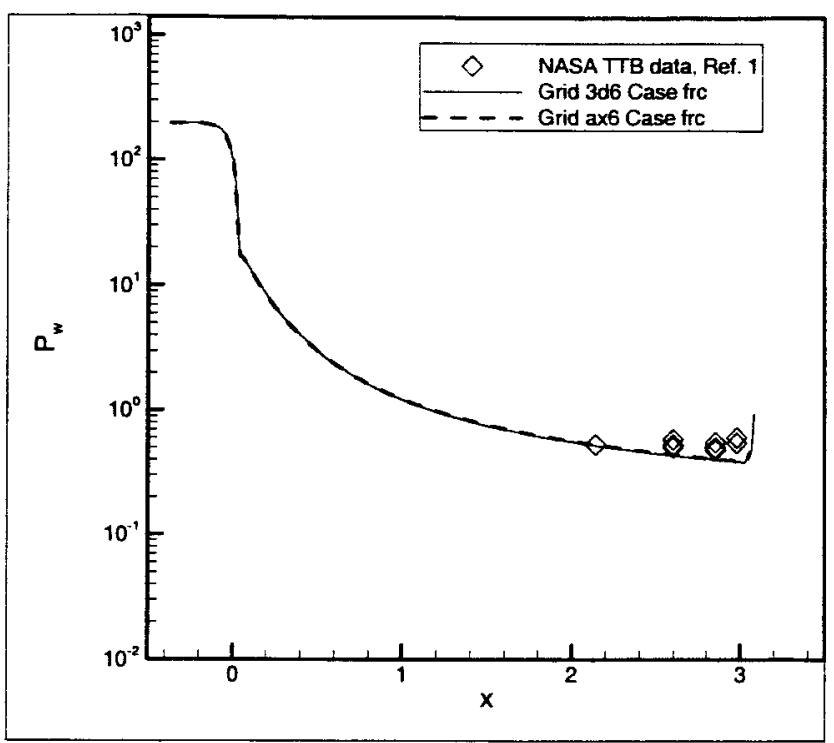

Fig. 15 A comparison of thruster wall pressures.

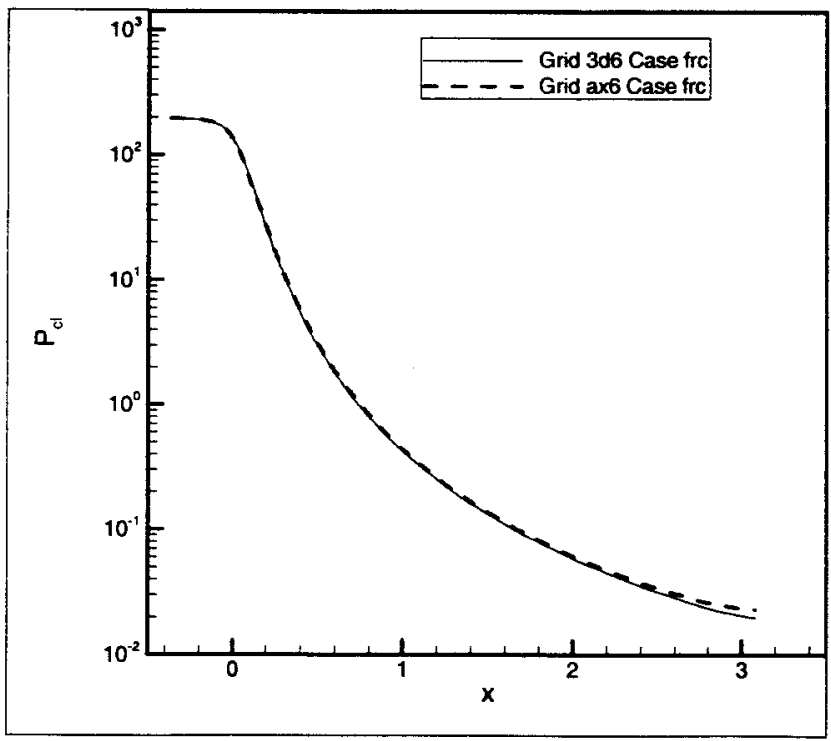

Fig. 16 A comparison of thruster centerline pressures. 


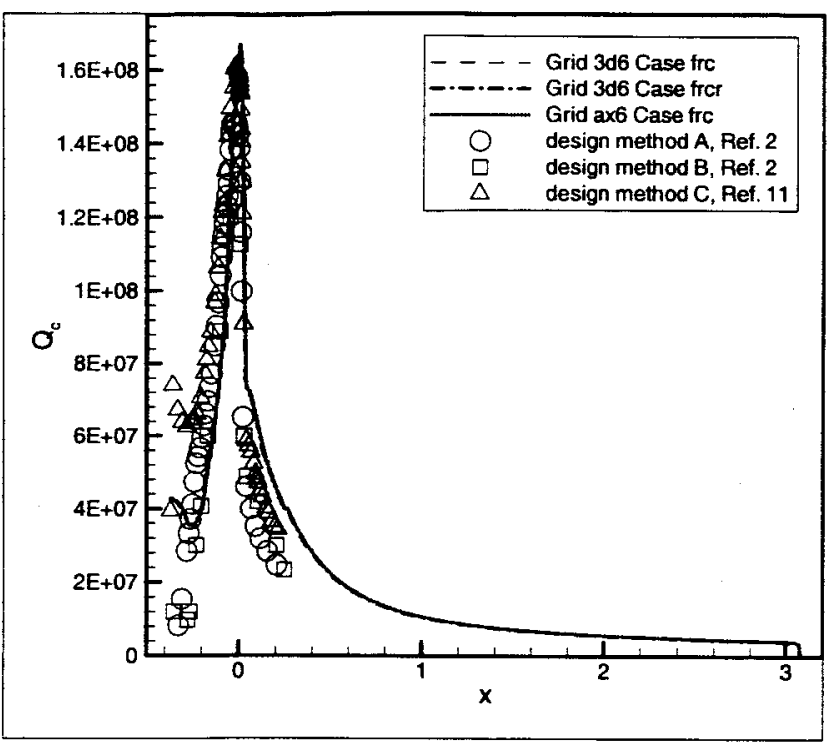

Fig. 17 A comparison of wall heat fluxes.

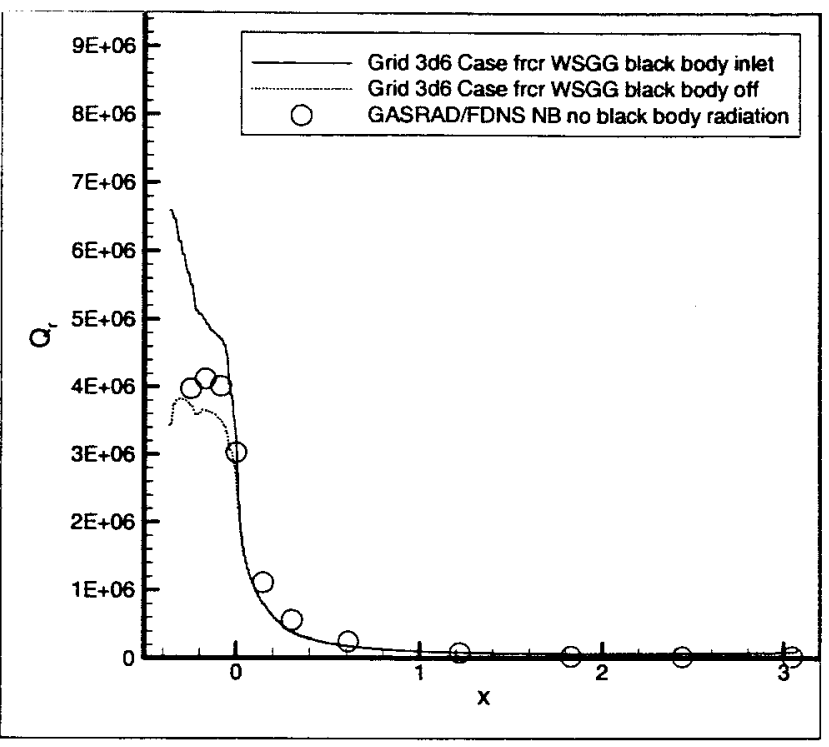

Fig. 18 A comparison of radiative wall heat fluxes. 